\title{
Impact of neoadjuvant chemotherapy on surgical outcomes among patients with hormone receptor positive breast cancer
}

\author{
Andrew N. Hage BS | Christina Capriccioso BS | Julia Brennan BS | \\ Brendan Heiden BS | Alexander Zheutlin BS | Michael S. Sabel MD (iD
}

Department of Surgery, University of Michigan Health Systems, Ann Arbor, Michigan

\section{Correspondence}

Michael S. Sabel, MD, 3302 Cancer Center, 1500 East Medical Center Drive, Ann Arbor, MI 48109.

Email: msabel@umich.edu
Background: Pathologic complete response ( $\mathrm{pCR}$ ) after neoadjuvant chemotherapy (NACT) is a surrogate for outcome, but not necessarily conversion to BCT eligibility. We sought to examine the impact of NACT on surgical decision making among HR+ patients.

Methods: Our IRB-approved breast cancer database was queried for patients who underwent NACT, including the clinicopathologic data and surgeon's pre- and postNACT assessment. Surgical conversion rate (SCR) was defined as patients ineligible for BCT prior to NACT, who were given the choice following NACT.

Results: Among 289 patients, pCR rates were highest among patients with HER2enriched subtype (60\%) and lowest in patients with luminal A disease (4\%). Overall, the BCT rate was $41 \%$, while $28 \%$ opted for bilateral mastectomy across subtypes. Despite a low pCR, the SCR was still high (54\%) among patients with the luminal A subtype. Conclusion: Despite poor pCR rates, NACT still has potential to improve surgical outcomes among hormone receptor positive patients. The surgical conversion rate is a superior measure of the impact of NACT on surgical decision making than examining $\mathrm{BCT}$ rates.

\section{KEYWORDS}

breast conservation, luminal $\mathrm{A}$, mastectomy, neoadjuvant chemotherapy

\section{1 | INTRODUCTION}

Neoadjuvant chemotherapy (NACT) has become a hallmark for the treatment of operable breast cancer by greatly expanding the pool of potential breast conserving therapy (BCT) candidates by downstaging tumors and permitting BCT in patients who would otherwise require a mastectomy. ${ }^{1-3}$ Response to NACT has also emerged as a prognostic sign. Patients with a pathologic complete response (pCR) to NACT have an improved overall survival. ${ }^{3,4}$ This has dramatically altered our approach to clinical trials of new therapeutics in breast cancer. By using the $\mathrm{pCR}$ rate as a surrogate marker for NACT efficacy, clinical trials can evaluate new therapeutic agents more rapidly than in the adjuvant setting.

The use of NACT has also broadened our understanding of tumor biology and differential response to treatment. Several studies have identified molecular subtypes, based on gene expression profiles that divide tumors into subgroups including luminal-like, basal-like, and Her-2/neu over-expressing and that these subgroups are associated with differences in outcome. ${ }^{5,6}$ As molecular profiling of each tumor is not feasible or cost-effective, clinicians use hormone receptor status, HER-2/neu expression, and either grade or $\mathrm{Ki}-67$ expression to group tumors into subtypes that approximate the molecular subtype signatures. ${ }^{7}$ These histologic subtypes, based on information routinely found in the pathology report from the diagnostic core biopsy, have also been shown to correlate strongly with the tumor's response to NACT, specifically the pCR rate.

The correlation between histologic subtype and response has allowed for a selective use of neoadjuvant chemotherapy. Patients with hormone receptor positive disease, particularly in the absence of Her-2/neu over-expression, have a low pCR rate. ${ }^{8}$ As such, there is 
often less enthusiasm for NACT on the basis that a pCR is less likely to be achieved. However, from a surgical perspective, a pCR is not necessary to permit BCT in a patient who would otherwise require a mastectomy. There is less data regarding the degree of downstaging based on histologic subtype. Most studies use the BCT rate as an endpoint, but this metric is skewed by multiple factors outside of response, and may not be reflective of the true rate of conversion from mastectomy to BCT. Herein, we sought to examine the percentage of patients converted from requiring a mastectomy to being eligible for $\mathrm{BCT}$, regardless of their ultimate surgery, based on histologic subtype.

\section{2 | METHODS}

All patients with biopsy-proven invasive breast cancer are presented at the University of Michigan Comprehensive Cancer Center's multidisciplinary tumor board composed of surgeons, medical and radiation oncologists, radiologists, pathologists, and associated support staff. Once the patient has completed surgery, the details of these discussions and the clinical and pathologic data are entered into our prospective breast cancer database. With Institutional Review Board approval, our database was queried for all adult female patients 21 years of age and older who underwent neoadjuvant chemotherapy prior to surgery at the University of Michigan between 2002 and 2014. Patients who had their pre-chemotherapy surgical evaluation at an outside institution, or surgery at an outside institution, were excluded from the study. Patients with inflammatory breast cancer or documented stage IV disease were excluded. Patients who had excision of the primary tumor prior to neoadjuvant therapy were also excluded.

The database was queried for the pre-chemotherapy radiologic and pathologic findings, operative details, and post-treatment pathologic findings. A detailed review of the multidisciplinary visit was conducted to determine the surgeon's recommendations; specifically, the suitability of patients to undergo BCT prior to neoadjuvant chemotherapy and whether the patient would be a eligible for BCT after neoadjuvant chemotherapy. Patients who were not suitable candidates for BCT prior to neoadjuvant chemotherapy, and had no contraindications to BCT after neoadjuvant chemotherapy (widespread calcifications, multi-centric disease, contraindication to radiation therapy), were classified as "Potential Downstage." The surgical conversion rate was defined as patients who were potential downstages who were given the option of BCT after neoadjuvant chemotherapy, regardless of the surgery they ultimately opted for. This also included patients felt to be BCT candidates, who ultimately underwent mastectomy secondary to inability to obtain negative margins.

Patients were categorized by histologic subtype based on estrogen receptor (ER), progesterone receptor (PR) and Her-2/neu expression using guidelines from the St. Gallen International Expert Consensus 2011 and 2013, splitting Luminal B into two groups (Luminal B- Her2- and Luminal B-like, Her2+) and using PR and Ki-67, with one exception. ${ }^{9}$ At the University of Michigan, Ki-67 expression is not routinely measured, so grade 3 was used in place of Ki-67 high. Patients who were ER positive, PR positive, and Her-2/neu negative, with grade 1 or grade 2 disease, were categorized as luminal $A$. Luminal B (Her2 neg) included patients who were ER positive, HER2 negative, and either grade 3 or PR low. Luminal B-like (Her2 pos) included patients who were ER positive, HER2 overexpressed and any grade or PR. HER2-enriched was defined as hormone receptor negative but overexpressing Her-2/neu, while basal-type was defined as triple negative.

\section{RESULTS}

After excluding patients with stage IV disease, inflammatory breast disease, and patients with no primary tumor in place prior to starting neoadjuvant chemotherapy, we identified 289 patients who had their pre-chemotherapy surgical assessment and subsequent surgery at the University of Michigan between 2002 and 2014. Median age was 49 (range 22-88). Two-hundred and thirty-two were Caucasian (80\%) while 34 (12\%) were African-American.

The pathologic features are summarized in Table 1 . The majority of cases were invasive ductal carcinoma (88\%), while $4 \%$ were lobular carcinoma. The average tumor size prior to chemotherapy was 4.12 , with $60 \%$ being $\mathrm{T} 2$ tumors, $30 \%$ being $\mathrm{T} 3$ and $10 \%$ being $\mathrm{T} 1$. Over half of the tumors, 163 , were grade 3 . Eighty-six $(30 \%)$ were grade 2 , while only $20(7 \%)$ were grade 1 . Another $21(7 \%)$ had unknown grades. Estrogen receptor (ER) was positive in $50 \%$ of cases, while PR was positive in $37 \%$. Her-2/neu was over-expressed in $33 \%$. Using hormone receptor status, Her-2/neu over-expression, and grade 3 as an alternative to Ki67, patients were stratified by histologic subtype. This resulted in 53 (18\%) tumors categorized as luminal A, 40 (14\%) categorized as Luminal B (Her2 neg), 53 (18\%) as Luminal B-like (Her2 pos), 43 (15\%) as HER2 enriched, and 100 (34\%) as basal type (triple negative).

Over this time period, pre-neoadjuvant chemotherapy nodal staging, using both needle biopsy and SLN biopsy was performed on the majority of patients. Needle biopsy, either FNA or core, was performed in 146 (50\%) of patients, while SLN biopsy was performed in 119 (41\%). Among the clinically node negative patients who underwent SLN biopsy prior to neoadjuvant chemotherapy, 48 (40\%) were positive while 71 (60\%) were negative. There were 25 patients (9\%) who did not have pathologic confirmation of their nodal status prior to neoadjuvant chemotherapy; 11 were clinically node positive while 14 were clinically node negative. Among the luminal A patients undergoing neoadjuvant chemotherapy, over half $(55 \%)$ were documented as node positive prior to chemotherapy. This was a lower percentage than the other subtypes; luminal B combined (71\%), HER2 enriched (71\%), and basal (60\%).

As expected, the complete pathologic response rate differed greatly by histologic subtype. Overall, the pCR rate was $32 \%$. Only four patients $(6 \%)$ with the luminal A subtype had a pCR. This was greater for the luminal B (30\%) and triple negative subtypes (40\%). The highest $\mathrm{pCR}$ rate was among patients with the HER2 enriched 
subtype at $62 \%$. Looking at the surgery that patients underwent at the completion of their chemotherapy, there is slightly less variation

TABLE 1 Clinical and pathologic features of patients receiving neoadjuvant chemotherapy

\begin{tabular}{|c|c|}
\hline \multicolumn{2}{|l|}{ Age } \\
\hline Mean & 48.9 years \\
\hline Range & $22-88$ \\
\hline \multicolumn{2}{|l|}{ Race } \\
\hline White & $232(80 \%)$ \\
\hline African-American & $34(12 \%)$ \\
\hline Asian & $10(3 \%)$ \\
\hline Island-Pacific & $3(1 \%)$ \\
\hline Hispanic & $3(1 \%)$ \\
\hline Other or unknown & $8(2 \%)$ \\
\hline \multicolumn{2}{|l|}{ Tumor size } \\
\hline $0-2 \mathrm{~cm}$ & $29(10 \%)$ \\
\hline $2-5 \mathrm{~cm}$ & $176(60 \%)$ \\
\hline$>5$ & $83(29 \%)$ \\
\hline Occult & $2(1 \%)$ \\
\hline \multicolumn{2}{|l|}{ Tumor histology } \\
\hline Ductal & $257(88 \%)$ \\
\hline Lobular & $12(4 \%)$ \\
\hline Other & $19(7 \%)$ \\
\hline Unknown/unclassified & $3(1 \%)$ \\
\hline \multicolumn{2}{|l|}{ Grade } \\
\hline 1 & $20(7 \%)$ \\
\hline 2 & $86(30 \%)$ \\
\hline 3 & $163(56 \%)$ \\
\hline Unknown & $21(7 \%)$ \\
\hline \multicolumn{2}{|l|}{ Nodal staging } \\
\hline Needle biopsy & $146(50 \%)$ \\
\hline Pre-NACT SLN Bx & 119 (41\%) \\
\hline No nodal staging & $25(9 \%)$ \\
\hline \multicolumn{2}{|c|}{ Nodal status prior to NACT } \\
\hline Positive(clin or path) & 199 (69\%) \\
\hline Path negative & $71(24 \%)$ \\
\hline Clinically negative & $20(7 \%)$ \\
\hline \multicolumn{2}{|l|}{ ER status } \\
\hline Negative & $144(50 \%)$ \\
\hline Positive & $144(50 \%)$ \\
\hline \multicolumn{2}{|l|}{ PR status } \\
\hline Negative & $108(37 \%)$ \\
\hline Positive & $182(63 \%)$ \\
\hline \multicolumn{2}{|l|}{ Her-2/neu status } \\
\hline Negative & 194 (67\%) \\
\hline Positive & 96 (33\%) \\
\hline
\end{tabular}

between the subtypes. The breast conservation therapy (BCT) rate overall was $41 \%$, while $28 \%$ of patients opted to undergo bilateral mastectomy. Table 2 breaks this down by subtype. Luminal A was associated with the lowest rate of BCT $(31 \%)$, while the triple negative patients had the highest (47\%). The decision to undergo bilateral mastectomy was evenly distributed across subtypes.

We further characterized the "surgical conversion rate" by eliminating patients who would or would not have been eligible for $\mathrm{BCT}$ from the onset, and determining whether patients were eligible for BCT after chemotherapy, regardless of what surgery they chose. These are shown in Figure 1. Among luminal A patients, 35 of 53 (66\%) were potential downstage candidates. Four patients were deemed eligible for BCT prior to neoadjuvant chemotherapy, while 14 patients had a contraindication to BCT prior to neoadjuvant chemotherapy (prior RT, multicentricity or widespread calcifications). Among these 46 patients who were not candidates for BCT prior to neoadjuvant (potential downstage), 16 patients were deemed poor candidates after neoadjuvant chemotherapy secondary to tumor size. There were 19 patients (54\%) who were converted to potentially BCT eligible. Among these patients, three opted for unilateral or bilateral mastectomies regardless, and two patients who were thought to be BCT eligible, ultimately underwent mastectomy based on pathology. Using the same formula, the surgical conversion rate was calculated for each of the subtypes. This surgical conversion rate was $66 \%$ for the luminal B (Her2 neg) patients, $83 \%$ for the luminal B-like (Her2 pos) patients, $85 \%$ for the HER2-enriched, and $78 \%$ for the basal subtypes.

With a mean follow-up of 5.84 years (three patients lost to followup), the local recurrence rate was $5 \% ; 2 \%$ for patients who had a complete pathologic response ( 2 of 90 , one luminal B-like one basal) and $7 \%$ for patients who did not. Among patients who did not have a $\mathrm{pCR}$, the local recurrence rate was highest for the HER2-enriched (2 of $15,12 \%)$ and basal (7 of $55,11 \%)$. The local recurrence rate was $4 \%$ for luminal A, $6 \%$ for Luminal B (Her2-), and $0 \%$ for Luminal B-like (Her-2+).

\section{DISCUSSION}

Neoadjuvant chemotherapy is the standard of care for patients with locally advanced or inflammatory breast cancer and a hallmark of therapy for women with operable breast cancer, allowing for a greater population of patients to undergo breast conservation. ${ }^{10,11}$ It has also allowed for downstaging of the axilla, allowing more women to avoid axillary lymph node dissection. ${ }^{12-14}$ Although the delivery of chemotherapy in the neoadjuvant setting has not demonstrated a definitive survival benefit over adjuvant chemotherapy, there are other potential advantages. One of these is the prognostic information derived from the response to NACT, in particular the achievement of a pathologic complete response ( $p C R$ ), which is now considered to be a surrogate marker of treatment efficacy. ${ }^{15}$ As such, many investigators are examining predictors of $\mathrm{pCR}$ which may allow for a better selection of patients. 
TABLE 2 Final surgery by histologic subtype

\begin{tabular}{|c|c|c|c|c|c|c|}
\hline & N (\%) & BCT & $\begin{array}{l}\text { Breast conservation rate } \\
\text { (\%) }\end{array}$ & $\begin{array}{l}\text { Unilateral } \\
\text { mastectomy }\end{array}$ & $\begin{array}{l}\text { Bilateral } \\
\text { mastectomy }\end{array}$ & $\begin{array}{l}\text { Bilateral mastectomy rate } \\
\text { (\%) }\end{array}$ \\
\hline Luminal A & $53(18)$ & 15 & 31 & 19 & 15 & 31 \\
\hline Luminal B (HER-) & $40(14)$ & 14 & 38 & 10 & 12 & 33 \\
\hline $\begin{array}{l}\text { Luminal B-like (HER2 } \\
\quad+)\end{array}$ & $53(18)$ & 20 & 37 & 18 & 15 & 28 \\
\hline HER2 enriched & $43(15)$ & 18 & 39 & 15 & 10 & 23 \\
\hline Basal & $\begin{array}{l}100 \\
(35)\end{array}$ & 47 & 47 & 23 & 25 & 26 \\
\hline Total & 289 & 114 & 41 & 85 & 77 & 28 \\
\hline
\end{tabular}

The predictive value of hormone receptor status, Her-2/neu overexpression and tumor grade have been well established, and in the absence of gene expression data, serve as surrogate classifications for breast cancer subtypes. ${ }^{7,16}$ Several groups have demonstrated clear differences in $\mathrm{pCR}$ rates by histologic subtype for breast cancer., ${ }^{8,17-21}$ While there are many studies looking at $\mathrm{PCR}$ rates by breast cancer subtype, there are only a few studies looking at the impact on surgical procedure. Boughey et $\mathrm{al}^{22}$ reported on the rates of BCT among patients on the American College of Surgeons Oncology Group (ACoSOG) Z1071 trial. They reported rates of BCT to be higher in patients with triple-negative and HER-2 positive breast cancer $(46.8 \%$ and $43.0 \%$, respectively) than inpatients with hormone-receptor positive, HER2-negative disease (34.5\%; $P=0.019)$. These remained significant predictors of breast conservation on multivariable analysis, along with older age, and lower tumor stage at presentation.

Simply measuring the percentage of patients who undergo lumpectomy after neoadjuvant chemotherapy is a poor marker of clinical response, as it does not reflect patients already eligible for BCT prior to neoadjuvant chemotherapy and patients who would never be eligible regardless of response. This is therefore an inaccurate measure of the impact of NACT on surgical decision making. In the Z1071 trial,
BCS was more common in patients who presented with T0-T2 tumors (52\%) than in T3-T4 tumors (23\%), which is not surprising as many of these patients were candidates for BCS prior to chemotherapy. ${ }^{22}$ This is impacted by histologic subtype as well. Patients with triple-negative or HER-2/neu overexpressing tumors with T0-T2 tumors are more likely to be considered for NACT, as chemotherapy is commonly recommended regardless of tumor size or nodal status, than HR +/HER-2- tumors, for whom chemotherapy may not be recommended.

In addition to not reflecting the fraction of patients who were not lumpectomy candidates prior to chemotherapy, simply measuring the $\mathrm{BCT}$ rate also discounts the increasing percentage of patients who opt for mastectomy after NACT, despite being down-staged. Increasingly, women are opting for unilateral and bilateral mastectomy as an alternative to BCT, and this continues to rise. For this reason, we performed a more detailed analysis of our experience with NACT among operable patients to measure the Surgical Conversion Rate; defined as the percentage of patients who were converted from requiring mastectomy to candidates for $\mathrm{BCT}$. This eliminates those patients who were eligible for BCT prior to NACT and those patients who were never going to be eligible (for example patients with prior

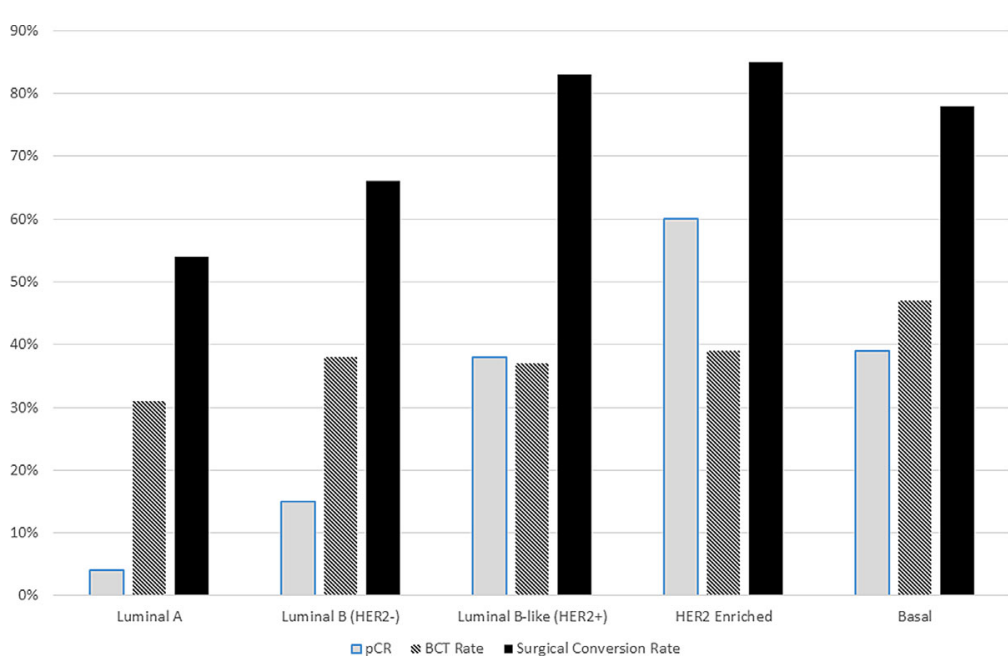

FIGURE 1 Pathologic and surgical response rates to neoadjuvant chemotherapy by histologic subtype 
radiation, multicentric disease or widespread calcifications). It also incorporates those patients who became eligible for BCT, but opted for mastectomy. This requires a documented surgical opinion both before and after chemotherapy. This data was often collected in the early trials of neoadjuvant chemotherapy, where a primary endpoint was breast conservation. However, as the $\mathrm{pCR}$ rate became the preeminent endpoint, collecting this data became increasingly less common.

This information, rather than $\mathrm{pCR}$, is critical to surgical decision making as converting a patient to BCT-eligible may be obtained with a partial response rather than a complete response. Partial response rate alone, however, is inadequate as a partial response does not guarantee BCS-eligibility. As opposed to pCR rates, the clinical response rates may be less dependent on histologic subtype. In the Z1071 data, changes in clinical tumor size between baseline and completion of NACT were relatively similar across histologic subtypes, with clinical partial responses in just over half of the patients. ${ }^{22}$ In our study, the surgical conversion rate tells a much different story that the $\mathrm{pCR}$ or $B C T$ rate. The luminal $A$ subtype had a significantly lower rate of $p C R$ (6\%), and the lowest rate of BCT (32\%). These numbers may suggest a lack of benefit to NACT in this population, but the surgical conversion rate was over $50 \%$. While this is lower than the luminal B (Her2 neg) (66\%) and considerably lower than luminal B-like (83\%) basal (78\%) and HER2 enriched (85\%\%), it demonstrates that a substantial number of patients with luminal A tumors realize a true clinical benefit from NACT despite the low likelihood of a pCR.

This does present a clinical catch-22 for patients who have large, clinically node-negative luminal A tumors that without down-staging would require a mastectomy. Despite the potential surgical benefit, chemotherapy decisions, in either the adjuvant or neoadjuvant setting, are based on preventing distant recurrence and improving survival. Many of these patients are not clear candidates for chemotherapy, and therefore, face a more complex decision algorithm. They could opt to move forward with mastectomy, and reserve chemotherapy choices until after the pathologic staging is complete, but many patients are highly motivated for breast preservation. Another option would be for neoadjuvant endocrine therapy. A meta-analysis of 20 randomized clinical trials involving almost 3500 patients demonstrated that neoadjuvant endocrine therapy was associated with response rates similar to neoadjuvant chemotherapy. ${ }^{23} \mathrm{~A}$ third choice, for patients motivated toward BCS, is to obtain more pre-NACT information. Sentinel lymph node biopsy prior to NACT, as was performed in several patients in this trial, is less commonly utilized, as post-NACT SLN biopsy more commonly allows for nodal down-staging and avoidance of ALND. Another option is the use of genomic assays to identify Luminal A patients who might be recommended for chemotherapy, and thus might opt to do this in the neoadjuvant setting. ${ }^{24}$ It is not clear whether these assays can predict response to chemotherapy, particularly with regard to surgical conversion, so more research in this regard is warranted. ${ }^{25}$

In conclusion, while a pCR is significantly less common among women with luminal A cancers, these patients still benefit from neoadjuvant chemotherapy in regards to downstaging and eligibility for breast conservation. Neoadjuvant chemotherapy is increasingly used among BCT-eligible patients, and more BCT-eligible patients are opting for unilateral or bilateral mastectomies. Therefore, BCT rates are an inaccurate measure of the impact of NACT on surgical therapy. Moving forward, clinical trials and outcome databases should prospectively record BCT-eligibility both pre- and post-neoadjuvant therapy so that the true surgical conversion rate, and associated predictive factors, can be truly assessed.

\section{REFERENCES}

1. Fisher B, Brown A, Mamounas E, et al. Effect of preoperative chemotherapy on local-regional disease in women with operable breast cancer: findings from National Surgical Adjuvant Breast and Bowel Project B-18. J Clin Oncol. 1997;15:2483-2493.

2. Baer HD, Anderson S, Smith RE, et al. Sequential preoperative or postoperative deocetaxel added to preoperative doxorubicin plus cyclophosphamide for operatibe breast cancer. NSABP Project Protocol B-27. J Clin Oncol. 2006;24:2019-2027.

3. Fisher B, Bryant J, Wolmark N, et al. Effect of preoperative chemotherapy on the outcome of women with operable breast cancer. J Clin Oncol. 1998;16:2672-2685.

4. Kong X, Moran MS, Zhang N, et al. Meta-analysis confirms achieving pathological complete response after neoadjvuant chemtoherapy predicts favourable prognosis for breast cancer patients. Eur J Cancer. 2084;47:2084-2090.

5. Sorlie T, Perou CM, Tibshirani R, et al. Gene expression patterns of breast carcinomas distinguish tumor subclasses with clinical implications. PNAS. 2001;98:10869-10874.

6. Perou CM, Sorlie T, Eisen MB, et al. Molecular portraits of human breast tumours. Nature. 2000;406:747-752.

7. Brenton JD, Carey LA, Ahmed AA, Caldas C. Molecular classification and molecular forecasting of breast cancer: ready for clinical application? J Clin Oncol. 2005;23:7350-7360.

8. Houssami N, Macaskill P, Von Minckwitz G, et al. Meta-analysis of the association of breast cancer subtype and pathologic complete response to neoadjuvant chemotherapy. Eur J Cancer. 2012;48:3342-3354.

9. Harbeck N, Thomssen C, Gnant M, Gallen ST. Brief preliminary summary of the consensus discussion. Breast Care. 2013;8:102-109.

10. Kaufmann M, von Minckwitz G, Smith R, et al. International expert panel on the use of primary (preoperative) systemic treatment of operable breast cancer: reveiw and recommendations. J Clin Oncol. 2003;21:2600-2608.

11. Loibl S, von Minckwitz G, Raab G, et al. Surgical procedures after neoadjuvant chemotherapy in operable breast cancer: results of the GEPARDUO trial. Ann Surg Oncol. 2006;13:1434-1442.

12. Mamtani A, Barrio AV, King TA, et al. How often does neoadjuvant chemotherapy avoid axillary dissection in patients with histologically confirmed nodal metastases? Results of a prospective study. Ann Surg Oncol. 2016;23:3467-3474.

13. Kelly AM, Dwamena B, Cronin P, Carlos RC. Breast cancer sentinel node identificationa nd classification after neoadjuvant chemotherapy -systematic review and meta analysis. Acad Radiol. 2009;16: 551-563.

14. Rouzier R, Extra JM, Klijanienko J, et al. Incidence and prognostic significance of complete axillary downstaging after primary chemotherapy in breast cancer patients wtih T1-T2 tumors and cytologically proven axillary metastatic lymph nodes. J Clin Oncol. 2002;20: 1304-1310.

15. Cortazar P, Zhang L, Untch M, et al. Pathological complete response and long-term clinical benefit in breast cancer: the CTNeoBCpooled analysis. Lancet. 2014;384:164-172.

16. Lips EH, Mulder L, de Ronde JJ, et al. Breast cancer subtyping by immunohistochemistry and histological grade outperforms breast 
cancer intrinsic subtypes in predicting neoadjuvant chemotherapy response. Breast Cancer Res Treat. 2013;140:63-71.

17. Colleoni $M$, Viale $G$, Zahrieh $D$, et al. Chemotherapy is more effective in patients with breast cancer not expressing steroid hormone receptors: a study of preoperative treatment. Clin Cancer Res. 2004;10:6622-6628.

18. Fisher ER, Wang J, Bryant J, et al. Pathobiology of preoperative chemotherapy findings from the national surgical adjuvant breast and bowel project (NSABP) protocol B-18. Cancer. 2002;95:681-695.

19. Faneyte IF, Schrama JG, Peterse JL, et al. Breast cancer response to neoadjuvant chemotherapy: predictive markers and relation with outcome. Br J Cancer. 2003;88:406-412.

20. Ring $A E$, Smith IE, Ashley $S$, et al. Oestrogen receptor status, pathological complete response and prognosis in patients receiving neoadjuvant chemotherapy for early breast cancer. Bri J Cancer. 2004;91:2012-2017.

21. Tan MC, Al Mushawah F, Gao F, et al. Predictors of complete pathological response after neoadjuvant systemc therapy for breast cancer. Am J Surgery. 2009;198:520-525.

22. Boughey JC, McCall LM, Ballman KV, et al. Tumor biology correlates with rates of breast-conserving surgery and pathologic complete response after neoadjuvant chemotherapy for breast cancer: findings from the ACOSOG Z1071 (Alliance) prospective multicenter clinical trial. Ann Surg. 2014;260:608-616.

23. Spring LM, Gupta A, Reynolds KL. Neoadjuvant endocrine therapy for estrogen receptor-positive breast cancer. JAMA Oncol. 2016;2: 1477-1486.

24. Cardoso F, van't Veer LJ, Bogaerts J, et al. 70-gene signature as an aid to treatment decisions in early-stage breast cancer. N Engl J Med. 2016;375:717-729.

25. Soran A, Bhargava R, Johnson R, et al. The impact of Oncotype DX recurrence score of paraffin-embedded core biopsy tissues in predicting response to neoadjuvant chemotherapy in women with breast cancer. Breast Dis. 2016;36:65-71.

How to cite this article: Hage AN, Capriccioso C, Brennan J, Heiden B, Zheutlin A, Sabel MS. Impact of neoadjuvant chemotherapy on surgical outcomes among patients with hormone receptor positive breast cancer. J Surg Oncol. 2017;116:665-670. https://doi.org/10.1002/jso.24721 